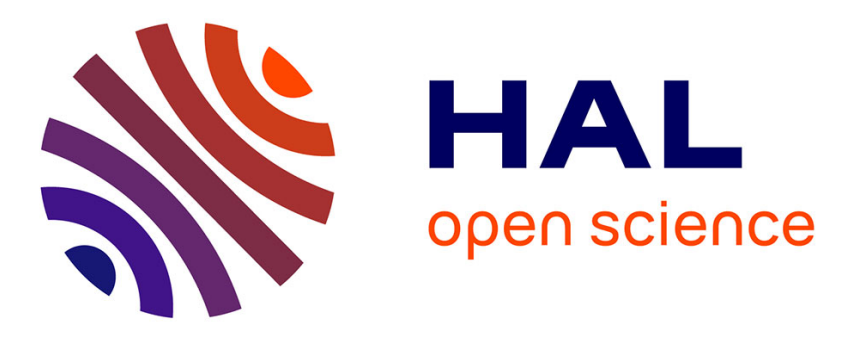

\title{
Modulation of hepatic malonyl-CoA/carnitine palmitoyltransferase 1A partnership creates a metabolic switch allowing oxidation of de novo fatty acids
}

Marie Akkaoui, Isabelle Cohen, Catherine Esnous, Véronique Lenoir, Martin

Sournac, Jean Girard, Carina Prip-Buus

\section{To cite this version:}

Marie Akkaoui, Isabelle Cohen, Catherine Esnous, Véronique Lenoir, Martin Sournac, et al.. Modulation of hepatic malonyl-CoA/carnitine palmitoyltransferase 1A partnership creates a metabolic switch allowing oxidation of de novo fatty acids. Biochemical Journal, 2009, 420 (3), pp.429-438. 10.1042/BJ20081932 . hal-00479100

\section{HAL Id: hal-00479100 https://hal.science/hal-00479100}

Submitted on 30 Apr 2010

HAL is a multi-disciplinary open access archive for the deposit and dissemination of scientific research documents, whether they are published or not. The documents may come from teaching and research institutions in France or abroad, or from public or private research centers.
L'archive ouverte pluridisciplinaire HAL, est destinée au dépôt et à la diffusion de documents scientifiques de niveau recherche, publiés ou non, émanant des établissements d'enseignement et de recherche français ou étrangers, des laboratoires publics ou privés. 


\section{Modulation of hepatic malonyl-CoA/carnitine palmitoyltransferase 1A partnership creates a metabolic switch allowing oxidation of de novo fatty acids \\ Marie AKKAOUI*†, Isabelle COHEN ${ }^{*} \uparrow$, Catherine ESNOUS $* \dagger$, Véronique LENOIR ${ }^{* \dagger} \uparrow$, Martin SOURNAC $* \dagger$, Jean GIRARD*† and Carina PRIP-BUUS*† \\ From the *Institut Cochin, Département d'Endocrinologie, Métabolisme et Cancer, Université Paris Descartes, CNRS Unité Mixte de Recherche 8104, and †INSERM U567, 24 rue du Faubourg Saint-Jacques, 75014 Paris, France}

Short Title : Modulation of liver malonyl-CoA/CPT1 partnership

Corresponding author : Carina Prip-Buus, Institut Cochin, Département d'Endocrinologie, Métabolisme et Cancer, Faculté de Médecine, 24 Rue du Faubourg Saint-Jacques, 75014 Paris, France. Tel : (33) 1537327 04; Fax : (33) 1444124 21; E-mail : carina.prip@inserm.fr

32

33 


\section{SYNOPSIS}

Liver mitochondrial $\beta$-oxidation of long-chain fatty acid (LCFA) is tightly regulated through inhibition of carnitine palmitoyltransferase 1A (CPT1A) by malonyl-CoA, an intermediate of lipogenesis stimulated by glucose and insulin. Moreover, CPT1A sensitivity to malonyl-CoA inhibition varies markedly depending on the physio-pathological state of the animal. Here, we asked whether an increase in CPT1A activity solely or in association with a decreased malonyl-CoA sensitivity could, even in the presence of high glucose and insulin concentrations, maintain a sustained LCFA ß-oxidation and/or protect from triglyceride accumulation in hepatocytes. We showed that adenovirus-mediated expression of rat wildtype CPT1A (CPT1wt) and malonyl-CoA-insensitive CPT1AM593S mutant (CPT1mt) in cultured fed rat hepatocytes counteracted the inhibition of oleate B-oxidation induced by 20 $\mathrm{mM}$ glucose/10 $\mathrm{nM}$ insulin. Interestingly, the glucose/insulin-induced cellular triglyceride accumulation was prevented, both in the presence and absence of exogenous oleate. This resulted from the generation of a metabolic switch allowing B-oxidation of de novo synthetised LCFA, which occured without alteration in glucose oxidation and glycogen synthesis. Moreover, CPT1mt expression was more effective than CPT1wt overexpression to counteract glucose/insulin effects, demonstrating that control of CPT1A activity by malonyl$\mathrm{CoA}$ is an essential driving force for hepatic LCFA metabolic fate. In conclusion, this study highlights that CPT1A is a prime target to increase hepatic LCFA $\beta$-oxidation and that acting directly on the degree of its malonyl-CoA sensitivity may be a relevant strategy to prevent and/or correct hepatic steatosis.

Key words: carnitine palmitoyltransferase 1, mitochondria, fatty acid oxidation, lipogenesis, triglyceride, liver 
61

62

63

64

65

66

67

68

69

70

71

72

73

74

75

76

77

78

79

80

81

82

83

84

85

86

87

88

89

90

91

92

93

94

95

96

97

98

99

100

101

102

103

104

105

106

107

108

109

110

\section{INTRODUCTION}

The hepatic isoform of carnitine palmitoyltransferase 1 (CPT1, EC 2.3.1.21) (CPT1A) is known to be the key regulatory enzyme in liver mitochondrial $\beta$-oxidation of long-chain fatty acids (LCFA) [1,2]. By converting long-chain acyl-CoA (LC-CoA) to acylcarnitine, CPT1A catalyzes the rate-limiting step in the entry of cytosolic LC-CoA into mitochondria where Boxidation takes place. Under physiological conditions, lipogenesis and LCFA B-oxidation are tightly regulated in the liver [1]. Malonyl-CoA, the first intermediate in lipogenesis, is synthetised by acetyl-CoA carboxylase (ACC) and is the substrate of fatty acid synthase (FAS) for de novo LCFA synthesis. Malonyl-CoA is also the physiological allosteric inhibitor of CPT1A [3]. Therefore, after feeding a carbohydrate-rich meal, the presence of both high plasma glucose and insulin concentrations stimulates liver glucose oxidation, glycogen storage and lipogenesis, allowing convertion of excess glucose to LCFA. The resulting increase in malonyl-CoA level inhibits CPT1A activity. Both exogenous LCFA taken up by the liver and endogenous LCFA generated by lipogenesis are then esterified into triglycerides (TG) and partly secreted as very-low-density lipoproteins (VLDL). Conversely, in the fasted state when lipogenesis is low, CPT1A is retrieved from malonyl-CoA inhibition. Hepatic Boxidation of LCFA released from adipose tissue can then occur to produce energy, cofactors required for optimal gluconeogenesis, and ketone bodies used as fuels by extrahepatic tissues $[1,2]$.

Disturbance of this key regulatory malonyl-CoA/CPT1A partnership might contribute to hepatic steatosis. Inherited CPT1A deficiency in humans is associated with hypoglycemia and hypoketonemia as well as hepatic steatosis during fasting [4,5]. Similarly to drug-induced impairment of mitochondrial ß-oxidation [6], pharmacological inhibition of CPT1A activity by irreversible inhibitors, such as tetradecylglycidic acid [7] or Etomoxir [8], induces mitochondrial injury leading to steatosis and inflammation. Whereas the consequences of a CPT1A activity defect are relatively known, the metabolic impact of an increased CPT1A activity per se had, until recently, never been reported in liver cells. During the meantime of our study was published that CPT1A overexpression in rat hepatocytes cultured in the presence of low glucose concentration increased LCFA B-oxidation capacity, leading to metabolic reorientation of exogenous LCFA taken up by the cells toward oxidation at the expense of esterification [9]. However, whether such an increased capacity to oxidize exogenous LCFA could be maintained in conditions under which this pathway is usually abolished, e.g. in the presence of high glucose and insulin concentrations known to stimulate de novo lipogenesis [10], had never been investigated in any model. Moreover, a unique feature of CPT1A is that its sensitivity to malonyl-CoA inhibition varies markedly depending on physiological state in adult rat. For example, it is increased by refeeding carbohydrate to fasted rats, by obesity state or after insulin administration to diabetic rats, whereas it is decreased by starvation and diabetes [11-15].

Thus, the aim of the present study was to clearly decipher whether a decrease in CPT1A malonyl-CoA sensitivity represents an efficient strategy to enhance mitochondrial LCFA Boxidation in liver cells. By overexpressing CPT1As with distinct malonyl-CoA sensitivity in cultured rat hepatocytes, we presently demonstrated that expression of a malonyl-CoAinsensitive CPT1A is more effective than overexpressing wild-type CPT1A to counteract glucose/insulin inhibitory effect on exogenous LCFA oxidation flux and to prevent glucose/insulin-induced TG accumulation. Moreover, modulation of the malonylCoA/CPT1A partnership generates a metabolic switch allowing ß-oxidation of de novo synthetised LCFA, hence preventing their esterification into TG. Altogether, these results highlight that control of CPT1A activity by malonyl-CoA is an essential driving force for hepatic LCFA metabolic fate and that acting directly on the degree of CPT1A malonyl-CoA 
111 sensitivity may be a relevant strategy to prevent and/or reduce liver steatosis.

112

113

114

115

116

117

118

119

120

121

122

123

124

125

126

127

128

129

130

131

132

133

134

135

136

137

138

139

140

141

142

143

144

145

146

147

148

149

150

151

152

153

154

155

156

157

158

159

160

\section{EXPERIMENTAL}

\section{Materials}

Collagenase used to isolate rat hepatocytes was purchased from Roche Diagnostics. Cell culture reagents (medium M199 with Earl salts, glutamine, Ultroser G) were obtained from Invitrogen. $\left[{ }^{14} \mathrm{C}\right]$ sodium bicarbonate was purchased from Perkin Elmer. $\left[1-{ }^{14} \mathrm{C}\right]$ oleate, D-[U$\left.{ }^{14} \mathrm{C}\right]$ glucose, L-[methyl- $\left.{ }^{3} \mathrm{H}\right]$ carnitine and $\left[1-{ }^{14} \mathrm{C}\right]$ acetate were purchased from GE Healthcare. 5-(Tetradecyloxy)-2-furoic acid (TOFA) was a gift from Dr. A. Richardson (Merrel National Laboratories, Cincinnati, OH). TLC silica-plates and dexamethasone were purchased from Merck Chemicals. Insulin was obtained from Novo Nordisk. Other biochemicals were purchased from Sigma-Aldrich.

\section{Construction of recombinant adenovirus}

The CPT1AM593S mutant (CPT1mt) was constructed with the QuikChange site-directed mutagenesis kit (Stratagene) using pYeDP1/8-10 containing the full-length rat CPT1A cDNA [16] as template, and the forward 5'CCTCACATATGAGGCCTCCAGTACCCGGCTCTTC CGAGAAGG-3' and reverse 5'CCTTCTCGGAAGAGCCGGGTACTGGAGGCCTCATAT GTGAGG-3' primers. Human adenovirus serotype 5 vectors (Ad) encoding either $\beta$ galactosidase (Ad-ßgal), wild-type rat CPT1A (Ad-CPT1wt) or CPT1mt (Ad-CPT1mt) under the control of the CMV promoter were produced by the Laboratoire de Thérapie Génique (INSERM U649, Nantes, France).

\section{Isolation of rat hepatocytes}

Male Wistar rats (200-300 g) were purchased from Elevage Janvier (France) and adapted to the environment for at least one week prior to the experiment. Rats were housed in plastic cages in a temperature-controlled $\left(21^{\circ} \mathrm{C}\right)$ and ventilated room with a $12 \mathrm{~h}$ light-dark cycle (light from 3 p.m. to 3 a.m.). They had free access to water and were fed ad libidum a standard rodent chow (A03, SAFE). All procedures were carried out according to the French guidelines for the care and use of experimental animals, and were approved by the Direction départementale des services vétérinaires de Paris. Hepatocytes were isolated at 9 a.m. by collagenase perfusion into the portal vein as described previously [10]. Isolated hepatocytes were resuspended in M199 medium containing $5 \mathrm{mM}$ glucose, penicillin $(100 \mathrm{UI} / \mathrm{ml})$, streptomycin $(100 \mu \mathrm{g} / \mathrm{ml}), 0.1 \%$ (w/v) BSA and $2 \mathrm{mM}$ glutamine (basal medium). From cell attachment, whatever the experiments presently performed, $1 \mathrm{mM}$ carnitine was systematically added to avoid a limitation in CPT1A activity which could alter LCFA oxidation. Cell viability estimated by tryptan blue exclusion was always greater than $80 \%$.

\section{Hepatocyte culture and adenovirus infection}

Hepatocytes were plated at a density of $8 \times 10^{6}$ cells $/ 100 \mathrm{~mm}$ petri dish (isolation of mitochondria), at $0.5 \times 10^{6}$ cells/well of 12 -well plates (MTS assay) or at $2.5 \times 10^{6}$ cells $/ 25$ $\mathrm{cm}^{2}$ flask (other experiments) and cultured for $4 \mathrm{~h}$ at $37^{\circ} \mathrm{C}$ in an incubator equilibrated with air $/ \mathrm{CO}_{2}(95: 5)$ in basal medium supplemented with $100 \mathrm{nM}$ dexamethasone, $10 \mathrm{nM}$ insulin and $2 \%(\mathrm{v} / \mathrm{v})$ Ultroser $\mathrm{G}$ before infection with adenovirus. After cell attachment, the medium was removed and hepatocytes were incubated for $2 \mathrm{~h}$ with $1 \mathrm{ml}$ (flask), $250 \mu 1$ (12-well plate) or $3 \mathrm{ml}$ (dish) of basal medium containing either $0,2.5$ or 5 infectious particles (ip)/cell of Ad-ßgal, Ad-CPT1wt or Ad-CPT1mt. Thereafter, the infection medium was removed and cells were further cultured for another $40 \mathrm{~h}$, the experiments being performed during the last 
161

162

163

164

165

166

167

168

169

170

171

172

173

174

175

176

177

178

179

180

181

182

183

184

185

186

187

188

189

190

191

192

193

194

195

196

197

198

199

200

201

202

203

204

205

206

207

208

209

210

$24 \mathrm{~h}$.

\section{Immunoblot analysis}

Aliquots of proteins were subjected to SDS/PAGE [17] in a 7\% gel and transferred onto nitrocellulose membrane. Detection of proteins was performed as described previously [16] using the ECL Pierce detection system (Perbio Sciences SAS). The antibodies used were against rat CPT1A [16], E. coli $\beta$-galactosidase (Rockland), rat FAS (gift from Dr. I. Dugail, INSERM U465, Paris, France), and human $\mathrm{ACC}_{2}$ (Upstate). For the generation of anti-CPT2 polyclonal antibody, peptide corresponding to the last $20 \mathrm{C}$-terminal residues of human CPT2 was synthesized, conjugated to keyhole limpet hemocyanin, and used to immunized New Zealand white rabbits (Neosystem). The immunoblots were quantified using a chemigenius apparatus (Syngene).

\section{Immunofluorescence assay}

Hepatocytes $\left(7 \times 10^{5}\right.$ cells/well of 6 -well plates) were cultured on coverslips coated with $4 \%$ $(\mathrm{v} / \mathrm{v})$ collagen in $0.001 \%(\mathrm{v} / \mathrm{v})$ acetic acid/PBS. Forty hours after infection (5 ip/cell), cells were fixed with $2 \%(\mathrm{v} / \mathrm{v})$ formaldehyde, permeabilized with $0.1 \%(\mathrm{v} / \mathrm{v})$ SDS and non-specific binding of antibodies was blocked by incubation with $10 \%(\mathrm{~V} / \mathrm{v}) \mathrm{SVF} / \mathrm{PBS}$ for $20 \mathrm{~min}$. Cells were stained with polyclonal anti-rat CPT1A and mouse monoclonal anti-cytochrome c (BD Biosciences) antibodies which were detected by a goat anti-rabbit IgG conjugated with Alexa ${ }^{\circledR}$ fluor 488 and a goat anti-mouse IgG conjugated with Alexa ${ }^{\circledR}$ fluor 594 (Molecular Probes), respectively. After incubation with $2 \mu \mathrm{M}$ Hoechst 33342 for DNA staining, coverslips were mounted on glass slides with fluoromount $G$ (Clinisciences). Fluorescent staining was viewed in a confocal laser-scanning microscope (Leica TCS SP2 AOBS). The captured images were processed using ImageJ software (Wayne Rasband).

\section{Isolation of mitochondria and CPT activity assay}

Forty hours after infection, cells were washed and scraped with ice-cold PBS. Mitochondria were isolated in an isolation buffer $(0.3 \mathrm{M}$ sucrose, $5 \mathrm{mM}$ Tris-HCl, $1 \mathrm{mM}$ EGTA, pH 7.4) using differential centrifugation [16]. Protein concentration was determined by Lowry method using BSA as a standard [18]. CPT1 activity was assayed in intact isolated mitochondria as palmitoyl-L-[methyl- $\left.{ }^{3} \mathrm{H}\right]$ carnitine formed from L-[methyl- $\left.{ }^{3} \mathrm{H}\right]$ carnitine $(400 \mu \mathrm{M} ; 10 \mathrm{Ci} / \mathrm{mol})$ and palmitoyl-CoA $(600 \mu \mathrm{M})$ in the presence of $1 \%(\mathrm{w} / \mathrm{v})$ BSA. Estimation of the malonyl$\mathrm{CoA} \mathrm{IC}_{50}$ value (concentration of malonyl-CoA required for $50 \%$ inhibition) and $\mathrm{CPT} 2$ activity were measured as described previously [16].

\section{Measurement of fatty acid metabolism}

During the last $24 \mathrm{~h}$ of culture, the medium was replaced by a medium containing either 5 $\mathrm{mM}$ glucose or $20 \mathrm{mM}$ glucose plus $10 \mathrm{nM}$ insulin. Fatty acid oxidation and esterification were determined during the last $2 \mathrm{~h}$ of culture in the presence of $0.3 \mathrm{mM}\left[1-{ }^{14} \mathrm{C}\right]$ oleate $(0.5$ $\mathrm{Ci} / \mathrm{mol}$ ) bound to $1 \%(\mathrm{w} / \mathrm{v})$ defatted BSA. After $2 \mathrm{~h}$ of incubation, medium was collected to determine $\left[{ }^{14} \mathrm{C}\right] \mathrm{CO}_{2},\left[{ }^{14} \mathrm{C}\right]$ acid-soluble products (ASP), unlabeled ketone body production (acetoacetate and $\beta$-hydroxybutyrate) and lactate and pyruvate concentrations. Cells were washed and scraped in PBS to determine $\left[{ }^{14} \mathrm{C}\right]$ triacylglycerol $(\mathrm{TG})$ and $\left[{ }^{14} \mathrm{C}\right]$ phospholipid (PL) production as previously described [19,20]. For the determination of very-low-density lipoprotein (VLDL) secretion, medium $(3 \mathrm{ml})$ was collected, immediately adjusted to $0.005 \%$ $(\mathrm{w} / \mathrm{v})$ gentamycin, $1 \mathrm{mM}$ EDTA, $0.04 \%(\mathrm{w} / \mathrm{v})$ sodium azide, and $0.02 \%(\mathrm{w} / \mathrm{v})$ sodium (ethylomercurithio)-2 benzoate, and was layered under $5 \mathrm{ml}$ of $0.15 \mathrm{M} \mathrm{NaCl}$. After centrifugation at $100000 \mathrm{~g}$ for $18 \mathrm{~h}$ at $10^{\circ} \mathrm{C}$, the $1 \mathrm{ml}$ top fraction containing VLDL was counted in $10 \mathrm{ml}$ scintillation liquid in a scintillation counter (Packard). 
212 Measurement of intracellular TG and Oil red $\mathrm{O}$ staining

213 For measurement of unlabeled TG, cellular lipids were extracted in chloroform/methanol (2:1, $214 \mathrm{v} / \mathrm{v}$ ) with vigorous shaking for $10 \mathrm{~min}$. After centrifugation for $25 \mathrm{~min}$ at $1200 \mathrm{~g}$, the lower 215 organic phase was collected, dried, and solubilized in chloroform:methanol. Lipid classes

216 were separated by TLC on silica-gel plates by using petroleum ether/diethyl ether/acetic acid $217(85: 15: 0.5, \mathrm{v} / \mathrm{v} / \mathrm{v})$ as the mobile phase. Lipids were visualized with $\mathrm{I}_{2}$ vapour. Bands were 218 scraped and TG were extracted from silica in chloroform:methanol. After centrifugation for $21945 \mathrm{~min}$ at $1500 \mathrm{~g}$ to precipitate silica, and evaporation, TG were measured with PAP 150 TG 220 kit (Biomerieux). Lipids droplets were detected in hepatocytes fixed with $3 \%(\mathrm{w} / \mathrm{v})$ 221 paraformaldehyde in PBS and colored with Oil red O [21].

222

223

224

225

226

227

228

229

230

231

232

233

234

235

236

237

238

239

240

\section{Measurement of ACC, FAS and malonyl-CoA decarboxylase (MCD) enzyme activity}

Cells were scrapped in ice cold $0.25 \mathrm{M}$ saccharose, $1 \mathrm{mM}$ DTT, $1 \mathrm{mM}$ EDTA and protease inhibitors, and cytosolic fractions obtained by differential centrifugation (10 min, $10000 \mathrm{~g}$, then $1 \mathrm{~h}, 100000 \mathrm{~g}$ at $4^{\circ} \mathrm{C}$ ) were used for ACC [22] and FAS activity [23] assay. MCD activity was assayed as described previously [24]

\section{Measurement of glucose metabolism}

During the last $24 \mathrm{~h}$ of culture, the medium was replaced by a medium containing either 5 $\mathrm{mM}$ glucose or $20 \mathrm{mM}$ glucose plus $10 \mathrm{nM}$ insulin in the absence or presence of $200 \mu \mathrm{M}$ TOFA, a specific ACC inhibitor [25]. Glucose oxidation and glycogen synthesis were determined during the last $2 \mathrm{~h}$ of culture in the presence of either $5 \mathrm{mM}\left[\mathrm{U}_{-}{ }^{14} \mathrm{C}\right] \mathrm{D}$-glucose $(133 \mu \mathrm{Ci} / \mathrm{mmol})$ or $20 \mathrm{mM}\left[\mathrm{U}-{ }^{14} \mathrm{C}\right] \mathrm{D}$-glucose $(87.5 \mu \mathrm{Ci} / \mathrm{mmol})$ plus $10 \mathrm{nM}$ insulin, without or with $0.3 \mathrm{mM}$ oleate bound to $1 \%(\mathrm{w} / \mathrm{v})$ defatted BSA. After $2 \mathrm{~h}$ of incubation, the medium was collected to determine $\left[{ }^{14} \mathrm{C}\right] \mathrm{CO}_{2}$ and unlabeled $\beta$-hydroxybutyrate production, whereas cells were washed three times with ice-cold PBS and scraped in $1 \mathrm{ml} 5 \mathrm{~N} \mathrm{KOH}$. Cold glycogen carrier $(2.5 \mathrm{mg})$ was added to the lysates $(600 \mu \mathrm{l})$ and samples were boiled for 30 min. Glycogen was precipitated overnight at $-20^{\circ} \mathrm{C}$ with two volumes of cold $100 \%$ ethanol. Precipitated glycogen was centrifuged at $10000 \mathrm{~g}$ for $10 \mathrm{~min}$. Pellets were washed once with $70 \%$ ethanol, resuspended in $0.5 \mathrm{ml}$ water, and counted by scintillation counting. Protein concentration was determined by Bradford method (BioRad) using BSA as a standard. Lipogenesis rate from $5 \mathrm{mM}\left[1-{ }^{14} \mathrm{C}\right]$ acetate $(10 \mu \mathrm{Ci}$ per flask) was determined during the last $2 \mathrm{~h}$ of culture as described previously [21]. Briefly, cells were rinsed twice with ice-cold PBS, immediately frozen in liquid nitrogen and scrapped off in $30 \%(\mathrm{v} / \mathrm{v}) \mathrm{KOH}$. Labeled fatty acids were then extracted and counted by scintillation counting.

\section{Measurement of mitochondrial activity}

MTS assay was performed in 12-well plates using the CellTiter 96 AQueous Non-Radioactive Cell Proliferation Assay (Promega) according to the manufacturer's instructions.

\section{Statistical analysis}

Results are expressed as means \pm S.E.M. Analysis of variance was carried out using StatView program (Abacus) to determine differences between groups. When significant differences significant difference (LSD) test $(\alpha=0.05)$. 
261

262

263

264

265

266

267

268

269

270

271

272

273

274

275

276

277

278

279

280

281

282

283

284

285

286

287

288

289

290

291

292

293

294

295

296

297

298

299

300

301

302

303

304

305

306

307

308

309

\section{RESULTS}

\section{Adenovirus-mediated expression of CPT1wt and CPT1mt in rat hepatocytes}

To investigate whether an increase in CPT1A activity solely or in association with a decreased malonyl-CoA sensitivity could modulate hepatic LCFA oxidation, primary rat hepatocytes were infected with 2.5 or $5 \mathrm{ip} /$ cell of adenovirus (Ad) encoding either wild-type rat CPT1A (CPT1wt) or mutant CPT1AM593S (CPT1mt) that is active but insensitive to malonyl-CoA inhibition [26-28]. This led to an increase in CPT1A protein expression in an adenovirus concentration dependent-manner in comparison to uninfected hepatocytes (Figure 1a). The protein level of another enzyme of the mitochondrial carnitine-shuttle system, CPT2, whose activity is insensitive to malonyl-CoA inhibition [1], was not modified (Figure 1a). As a control, hepatocytes were also infected with an adenovirus encoding $\beta$-galactosidase ( $\beta$ gal). The resulting $\beta$ gal expression had no repercussion on CPT1A and CPT2 protein levels when compared to uninfected cells (Figure 1a). Considering these results, the amount of $5 \mathrm{ip} / \mathrm{cell}$ was chosen for subsequent infection experiments. Immunofluorescence analysis of infected hepatocytes showed that both overexpressed CPT1wt and CPT1 $1 \mathrm{mt}$ proteins were colocalized with cytochrome c, a mitochondrial marker protein (Figure 1b), indicating as expected a mitochondrial localization. Moreover, infection with Ad-CPT1wt and Ad-CPT1mt respectively led to a 15 - and 11-fold increase in mitochondrial CPT1A protein level and a 7and 3-fold increase in CPT1A activity when compared to mitochondria isolated from either Ad-3gal-infected or uninfected hepatocytes (Figure 2a). In agreement with Figure 1a, CPT1wt and CPT1mt overexpression had no repercussion on both mitochondrial CPT2 protein level and activity (Figure 2a).

To evaluate the capacity of CPT1mt to exhibit enzyme activity despite the presence of malonyl-CoA, malonyl-CoA sensitivity was then measured in isolated mitochondria. In uninfected hepatocytes and Ad-Bgal- or Ad-CPT1wt-infected hepatocytes, CPT activity was almost completely suppressed $(80 \%)$ by the highest malonyl-CoA concentration used (150 $\mu \mathrm{M})$ (Figure 2b). This was indicative of a good membrane integrity of the isolated mitochondria and suggested that only CPT1A activity was measured without any significant contribution of CPT2. Their corresponding $\mathrm{IC}_{50}$ values for malonyl-CoA were not significantly different (uninfected : $2.5 \pm 0.7 \mu \mathrm{M}$; Ad- $\beta$ gal : $2.4 \pm 0.6 \mu \mathrm{M}$; Ad-CPT1wt : 12.2 $\pm 8.7 \mu \mathrm{M})$. By contrast, in Ad-CPT1mt-infected hepatocytes, CPT1A activity was not inhibited by 1 to $10 \mu \mathrm{M}$ of malonyl-CoA (Figure 2b). Moreover, even in the presence of 150 $\mu \mathrm{M}$ of malonyl-CoA, only $43 \%$ of the activity was inhibited which likely corresponds to the inhibition of endogenous CPT1A. Altogether, these results confirm that CPT1wt and CPT1mt overexpression in rat hepatocytes increases mitochondrial CPT1A protein level and activity, CPT1mt being insensitive to malonyl-CoA inhibition.

\section{CPT1wt and CPT1mt overexpression counteracts glucose and insulin effects on lipid metabolism}

CPT1wt and CPT1mt overexpression in cultured rat hepatocytes increased LCFA B-oxidation capacity under basal conditions of culture (5 $\mathrm{mM}$ glucose), leading to metabolic reorientation of exogenous LCFA toward oxidation at the expense of esterification (Supplementary Data 1). We then evaluated their metabolic effects in the presence of high concentrations of glucose and insulin, conditions known to promote hepatic lipogenesis [10], hence preventing mitochondrial LCFA oxidation [1]. In uninfected hepatocytes, a 24 h-exposure to $20 \mathrm{mM}$ glucose plus $10 \mathrm{nM}$ insulin markedly stimulated the expression and the activity of lipogenic ACC and FAS enzymes (Supplementary Data 2a-c), de novo lipogenesis (see below Figure 6b) and suppressed $\left[1-{ }^{14} \mathrm{C}\right]$ oleate oxidation by more than $90 \%$ (Figure 3a,b). Similar results 
310 were obtained in Ad-ßgal-infected hepatocytes. Overexpression of CPT1wt and CPT1mt did 311 not alter the glucose/insulin-stimulated expression and activity of ACC and FAS, nor the 312 enzymatic activity of malonyl-CoA decarboxylase (MCD) that catalyses the degradation of 313 malonyl-CoA into acetyl-CoA (Supplementary Data 2). However, their overexpression led to 314 a marked increase in $\left[1-{ }^{14} \mathrm{C}\right]$ oleate oxidation. Indeed, $\left[{ }^{14} \mathrm{C}\right] \mathrm{CO}_{2}$ and $\left[{ }^{14} \mathrm{C}\right]$ acid-soluble product 315 (ASP) productions were respectively increased by 4- and 6.8-fold for Ad-CPT1wt-infected 316 hepatocytes and by 6.6- and 13.6-fold for Ad-CPT1mt-infected hepatocytes, when compared 317 to Ad- $\beta$ gal-infected hepatocytes (Figure 3a,b). Conversely, only CPT1mt expression 318 significantly decreased by $44 \%\left[1-{ }^{14} \mathrm{C}\right]$ oleate esterification into TG when compared to Ad$319 \quad$ Bgal-infected cells (Figure 3c), without any significant modification of its esterification into phospholipids (PL) (Figure 3d). In the presence of glucose and insulin, the total amount of [1${ }^{14} \mathrm{C}$ ]oleate metabolized was similar for all infections (uninfected : $103 \pm 21$; Ad- $\beta$ gal : $104 \pm$ 18; Ad-CPT1wt : $115 \pm 12$ and Ad-CPT1mt : $138 \pm 17 \mathrm{nmol} / 2 \mathrm{~h} / \mathrm{mg}$ of prot). Whereas [1$\left.{ }^{14} \mathrm{C}\right]$ oleate oxidation rate represented only $5 \%$ of $\left[{ }^{14} \mathrm{C}\right]$ oleate metabolized in Ad- $\beta$ gal-infected hepatocytes, CPT1wt and CPT1mt overexpression respectively increased this rate up to $30 \%$ and $47 \%$ (Figure $3 \mathrm{e}$ ). To determine whether the decrease in $\left[{ }^{14} \mathrm{C}\right] \mathrm{TG}$ production, and hence in esterification flux, was not due to an increased export of TG as VLDL, $\left[1-{ }^{14} \mathrm{C}\right]$ oleate incorporation into VLDL secreted into the culture medium was also measured. $\left[1-{ }^{14} \mathrm{C}\right] \mathrm{VLDL}$ secretion was increased by $50 \%$ and $80 \%$ in hepatocytes overexpressing CPT1wt and CPT1mt, respectively (Figure 3f). However, the corresponding increased amount of incorporated oleate into VLDL ( 1.5 and $1.8 \mathrm{nmol} / 2 \mathrm{~h} / \mathrm{mg}$ of prot) was 10 - and 20 -fold lower than the observed reduction in oleate incorporation into TG (18 and $35 \mathrm{nmol} / 2 \mathrm{~h} / \mathrm{mg}$ of prot). Consequently, oleate incorporated into VLDL can likely explain no more than $10 \%$ of the decrease in TG esterification. Thus, in the presence of glucose and insulin, CPT1A overexpression decreased exogenous oleate esterification into TG by enhancing hepatic LCFA oxidation capacity, the effects of CPT1mt expression being always significantly higher than those observed for CPT1wt.

To determine whether this decrease in exogenous oleate esterification was reflected in intracellular lipid content, Oil red O staining of hepatocytes, which allows visualization of neutral lipids within the cells, and measurement of intracellular TG content were performed. A 24 h-exposure to glucose and insulin induced in Ad- $\beta$ gal-infected cells a huge increase in lipid droplets, which was much lower in hepatocytes expressing CPT1mt (Figure 4a). Moreover, whereas the presence of glucose and insulin induced a 5-fold increase in TG content both in uninfected and Ad- $\beta$ gal-infected hepatocytes, CPT1mt expression decreased the TG content by $43 \%$ when compared to Ad- $\beta$ gal-infected hepatocytes (Figure $4 \mathrm{~b}$, left panel). CPT1wt overexpression led to an intermediate decrease in TG content (Figure 4b, left panel). In these culture conditions, cellular TG could result from esterification of both LCFA produced by de novo lipogenesis and exogenous oleate. To determine the contribution of TG coming from lipogenesis, intracellular TG were quantified after a $24 \mathrm{~h}$-treatment with glucose and insulin, but in the absence of oleate. In these conditions, the TG content was also markedly increased in both uninfected and Ad- $\beta$ gal-infected hepatocytes (Figure $4 \mathrm{~b}$, right panel). CPT1wt and CPT1mt overexpression led to a respective $25 \%$ and $50 \%$ decrease in the glucose/insulin-stimulated TG accumulation when compared to Ad- $\beta$ gal-infected hepatocytes, with a significant difference between CPT1wt and CPT1mt (Figure 4b, right panel). Whatever the presence or not of exogenous oleate, the TG accumulation induced by glucose and insulin was not statistically different in hepatocytes infected with Ad- $\beta$ gal (Figure 4b). This indicated that accumulated cellular TG mostly resulted from esterification of LCFA newly synthetised from glucose. Thus, the increased capacity for LCFA oxidation due to CPT1wt and CPT1mt overexpression counteracts the glucose/insulin-induced accumulation of 
TG coming from lipogenesis.

\section{Effect of CPT1wt and CPT1mt overexpression on glucose metabolism}

To understand how the level of cellular TG resulting from lipogenesis could be decreased, two hypothesis were explored. The first one was that CPT1wt and CPT1mt overexpression could induce a decrease in glucose utilization, leading to a lower cellular TG content. To answer this question, $\left[{ }^{14} \mathrm{C}\right]$ glucose oxidation and $\left[{ }^{14} \mathrm{C}\right]$ glycogen synthesis were measured during the last $2 \mathrm{~h}$ of culture in the absence of exogenous oleate. As expected, glucose and insulin markedly stimulated glucose oxidation into $\mathrm{CO}_{2}$ and glycogen synthesis (Figure 5a,b, left panel). Infection of hepatocytes with either Ad-ßgal, Ad-CPT1wt or Ad-CPT1mt had no significant effect on $\left[{ }^{14} \mathrm{C}\right] \mathrm{CO}_{2}$ production and $\left[{ }^{14} \mathrm{C}\right]$ glycogen synthesis (Figure 5a,b, left panels). A trend of decreased $\left[{ }^{14} \mathrm{C}\right] \mathrm{CO}_{2}$ production was observed in hepatocytes infected with Ad-CPT1wt or Ad-CPT1mt. However, pyruvate, lactate, malate and oxaloacetate levels were not altered by CPT1wt and CPT1mt overexpression (data not shown). These results suggested that, in our experimental conditions, CPT1wt and CPT1mt overexpression did not alter glucose utilization. Similar results were observed in the presence of oleate (Figure 5a,b, right panels), indicating that, even when LCFA oxidation flux was further enhanced by addition of exogenous LCFA, glucose metabolism was also not altered.

The second hypothesis was that CPT1wt and CPT1mt overexpression, which leads to an increase in LCFA oxidation capacity, might allow the oxidation of LCFA newly synthetised from glucose. To test this hypothesis, ß-hydroxybutyrate production was measured in hepatocytes cultured in the absence or presence of $0.3 \mathrm{mM}$ oleate during the last $2 \mathrm{~h}$ of culture. As expected, in basal conditions of culture ( $5 \mathrm{mM}$ glucose) and in the absence of exogenous oleate, $\beta$-hydroxybutyrate level remained low in uninfected hepatocytes whereas it increased by 2.8 -fold upon oleate addition (Figure 6a). In uninfected and Ad-ßgal-infected hepatocytes, a 24 h-exposure to glucose and insulin decreased $\beta$-hydroxybutyrate production to the same basal level whatever the presence or not of oleate (Figure 6a). By contrast, in the presence of oleate, $\beta$-hydroxybutyrate level respectively increased by 2.5 - and 3.2-fold in CPT1wt and CPT1mt overexpressing hepatocytes, hence abrogating the glucose/insulin inhibitory effect on ketogenesis (Figure 6a, right panel). The observation that a 1.7-fold (CPT1wt) and 2.3-fold (CPT1mt) increase in B-hydroxybutyrate production occurred in the absence of exogenous oleate (Figure 6a, left panel) strongly suggested that LCFA arising from lipogenesis are oxidized to produce ketone bodies. To confirm this, hepatocytes were cultured for $24 \mathrm{~h}$ in the presence of TOFA, a specific ACC inhibitor [25]. As expected, TOFA treatment of uninfected hepatocytes totally inhibited both basal and glucose/insulin-stimulated de novo lipogenesis (Figure 6b) as well as glucose/insulin-induced TG accumulation (Figure $6 \mathrm{c})$. In these conditions, the increase in $\beta$-hydroxybutyrate production previously observed in CPT1wt and CPT1 $1 \mathrm{mt}$ overexpressing hepatocytes was fully abrogated (Figure 6a), confirming that the produced $\beta$-hydroxybutyrate arised from the oxidation of de novo synthetised LCFA. Thus, the higher induced increase in $\beta$-hydroxybutyrate production observed in the presence of exogenous oleate (Figure 6a) resulted from the oxidation of both exogenous and de novo synthetised LCFA. As observed previously, CPT1mt expression had, both in the absence and presence of oleate, a significantly higher effect than CPT1wt overexpression. Altogether, these results indicate that the observed decrease in cellular TG content in the absence of exogenous LCFA (Figure $4 \mathrm{~b}$ ) is not due to an alteration in glucose utilization following CPT1wt or CPT1mt overexpression, but results from the oxidation of LCFA newly synthetised from glucose. 
408

409

410

411

412

413

414

415

416

417

418

419

420

421

422

423

424

425

426

427

428

429

430

431

432

433

434

435

436

437

438

439

440

441

442

443

444

445

446

447

448

449

450

451

452

453

454

455

456

457

\section{DISCUSSION}

The present study provides the first proof of concept that, even in conditions under which lipogenesis is stimulated, a direct modulation of the malonyl-CoA/CPT1A partnership in liver cells is a relevant strategy to maintain a high mitochondrial LCFA B-oxidation flux which protects against TG accumulation.

In agreement with previous in vitro studies performed in pancreatic $\beta$-cells [27], muscle cells [29] and hepatocytes [9], the present study showed that overexpression of rat CPT1wt or expression of CPT1mt in cultured rat hepatocytes increased LCFA ß-oxidation capacity under basal conditions of culture. To challenge whether this increased oxidative capacity could be maintained in conditions under which LCFA B-oxidation is usually abolished, we chose to expose primary cultured rat hepatocytes to $20 \mathrm{mM}$ glucose plus $10 \mathrm{nM}$ insulin, conditions known to elicit hepatic lipogenesis [10]. As expected, this markedly increased glucose oxidation and glycogen synthesis, stimulated ACC and FAS expression and enzymatic activity as well as de novo lipogenesis, and led to TG accumulation, whereas it abolished exogenous oleate oxidation and ketogenesis. We presently showed that CPT1A overexpression partially (CPT1wt) or fully (CPT1mt) abolished the inhibitory effect of glucose/insulin on oleate oxidation. This allowed, in the case of CPT1mt expression, the maintenance of an oxidation flux and a ketone body production from exogenous LCFA similar to those observed in basal conditions of culture. It is noteworthy that this was accomplished without alteration in the stimulatory effects of glucose/insulin on lipogenic enzymes expression and activity and on glucose utilization. Moreover, the higher effectiveness of CPT1mt than CPT1wt overexpression to counteract the glucose/insulin effects on hepatic lipid metabolism occurred despite a lower increase in both mitochondrial CPT1A protein level and activity. The biological relevance of this finding is that it represents the converse experimental demonstration of McGarry's discovery of the physiological role of malonyl-CoA in controlling hepatic LCFA metabolic fate [1-3]. Although liver mitochondrial glycerol-3-phosphate acyltransferase 1 isoform, which catalyzes the first step in glycerolipid synthesis, may be important for LCFA metabolic channeling by competing with CPT1A for cytosolic LC-CoAs [30,31], our data clearly demonstrated that control of CPT1A activity by malonyl-CoA is an essential driving force for exogenous LCFA ß-oxidation flux. Additionally, they strengthened our prior findings that changes in the degree of CPT1A malonyl-CoA sensitivity, which occur in vivo in different physiopathological situations [1115], play a critical role in the regulation of hepatic LCFA oxidation flux [19,32]. Therefore, this observation let us to predict that strategies aiming to increase only CPT1A activity might be limited in situations where malonyl-CoA levels remain high. This may explain why a compensatory increase in CPT1 A gene expression might be insufficient to prevent hepatic TG accumulation when lipogenesis is exacerbated such as in mice overexpressing acylCoA:diacylglycerol acyltransferase 2 in the liver [33].

The key finding of our study is that, in the absence of exogenous LCFA, CPT1mt expression still markedly protects liver cells from glucose/insulin-induced TG accumulation. In these culture conditions, TG can only be produced by esterification of LCFA de novo synthetised from glucose through lipogenesis. By deciphering the metabolic impact of CPT1mt expression, we clearly showed that the decreased TG content directly resulted from $\beta$-oxidation of the newly synthetised LCFA as reflected by the increased $\beta$-hydroxybutyrate production. Thus, an increased CPT1A activity in association with a decreased malonyl-CoA sensitivity allowed hepatocytes to oxidize LCFA whatever their origin i.e. exogenous or endogenous (Figure 7). According to the Randle's glucose/fatty acid cycle, one would predict that increased LCFA B-oxidation may have an effect on the metabolic fate of glucose [34]. However, previous studies reported that CPT1wt and CPT1mt overexpression did not affect 
glucose metabolism in pancreatic B-cells [27] and in muscular cells [28]. Similarly, in the present study, an increased LCFA $\beta$-oxidation flux in hepatocytes did not altered the insulininduced glucose oxidation and glycogen synthesis. Additionally, oxidation of both glucose and LCFA is associated with conversion of oxidized cofactors into reduced cofactors which are then re-oxidized by the mitochondrial respiratory chain in order to permit other cycles of fuel oxidation [35]. The lactate/pyruvate and $\beta$-hydroxybutyrate/acetoacetate ratio respectively reflect the cytosolic and mitochondrial NADH/NAD ${ }^{+}$ratio. In the presence of glucose plus insulin, CPT1wt and CPT1mt overexpression did not modify the lactate/pyruvate ratio, suggesting no alteration in the cytosolic redox state. $\beta$-hydroxybutyrate/acetoacetate ratio increased upon CPT1wt and $\mathrm{CPT} 1 \mathrm{mt}$ overexpression but did not exceed the one measured in basal conditions of culture (data not shown). Moreover, the mitochondrial activity reflected by a MTS assay was not modified by CPT1wt and CPT1mt overexpression (Supplementary Data 3). This suggests that mitochondria, in our experimental conditions, are able to handle efficiently the re-oxidation of NADH when both glucose and LCFA are oxidized. As illustrated in the present study, the fundamental role of the hepatic malonyl$\mathrm{CoA} / \mathrm{CPT} 1 \mathrm{~A}$ partnership is to provide a powerful regulatory mechanism for metabolic switch between carbohydrate and lipid utilization as energy fuel. Presently, we report for the first time that the malonyl-CoA/CPT1A interaction is also essential to preserve the cells from wasting energy by diverting the newly de novo synthetised LCFA from mitochondrial oxidation (Figure 7). This may greatly contribute to the physiological function of the liver to convert excess dietary carbohydrates into TG.

Because hepatic steatosis is a risk factor for nonalcoholic steatohepatitis and type 2 diabetes, research efforts have focused on a decreased capacity for de novo lipogenesis in an attempt to prevent and/or reduce hepatic steatosis. Among them, liver-specific suppression of rat ACC isoforms expression [36], inhibition of the nuclear factor carbohydrate responsive element binding protein gene expression [37], which decreased ACC and FAS gene expression, and hepatic MCD overexpression [38] were effective in decreasing TG content in fed animals and/or hepatic steatosis and insulin resistance in obese animals. Due to the existence of the malonyl-CoA/CPT1A partnership, these strategies also stimulated LCFA Boxidation flux through the release of CPT1A from malonyl-CoA inhibition [36-38]. Direct evidence that an enhanced capacity to oxidize LCFA per se, independently of a reduced lipogenesis flux, may contribute to decrease liver TG content was recently provided by in vivo overexpression of wild-type CPT1A in rat liver [9]. However, in high-fat diet-induced obese rats, hepatic CPT1A overexpression led only to a slight increase in LCFA ß-oxidation capacity and a substantial reduction in hepatic TG accumulation whereas no improvement of insulin sensitivity could be observed [9]. Such moderate effects might be due to the presence of elevated malonyl-CoA concentration which, despite an increased CPT1A expression, can limit in vivo CPT1A activity since the latter remains malonyl-CoA sensitive. Therefore, we propose that, in addition to an increase in CPT1A expression, acting directly on the degree of CPT1A malonyl-CoA sensitivity, might be a more relevant strategy in situations of insulin resistance which are associated with an increase in LCFA delivery to the liver (increased consumption of a high-fat diet and/or lipolysis) and in endogenous LCFA (excessive lipogenesis).

In summary, a direct modulation of malonyl-CoA/CPT1A interaction in liver cells, independently of the lipogenic pathway, allows mitochondrial oxidation of both exogenous LCFA taken up by the liver and endogenous LCFA generated by lipogenesis, without alteration in glucose utilization. Thus, whereas CPT1A inhibition by malonyl-CoA is essential to avoid in physiological situations a waste of energy, modulation of its malonyl-CoA sensitivity may be a useful therapeutic strategy to prevent and/or reduce liver steatosis. 


\section{ACKNOWLEDGMENTS}

511 This manuscript is dedicated to Pr. Denis J. McGarry. We thank France Demaugre (INSERM 512 U785, Villejuif, France) and Abdelhak Mansouri for critical review of the manuscript, and 513 Sylvie Demignot (CNRS UMR 505, Paris, France) for helpful suggestions for TG and VLDL 514 measurements. We thank the Vector Core of the University Hospital of Nantes supported by 515 the Association Française contre les Myopathies (AFM) for providing the adenovirus vectors.

\section{FUNDING}

520

\section{REFERENCES}

530

5311 McGarry, J. D. and Brown, N. F. (1997) The mitochondrial carnitine 532

533

534

535

536

537

Marie Akkaoui is a recipient of a MENRT (Ministère de l'Education Nationale, de la Recherche et de la Technologie) fellowship. This work was supported in part by research grants from the Ministère de la Recherche "ACI Biologie du Développement et Physiologie Intégrative" [0220527], ALFEDIAM-AstraZeneca, INSERM "Programme National de Recherches sur le Diabète" [ASE04179KSA] and the Agence Nationale de la Recherche "Cardiovasculaire, Obésité, Diabète" [APV05051KSA].

543

544

545

546

547

548 palmitoyltransferase system. From concept to molecular analysis. Eur J Biochem 244, $1-14$

2 Zammit, V. A. (2008) Carnitine palmitoyltransferase 1: central to cell function. IUBMB Life 60, 347-354

3 McGarry, J. D., Mannaerts, G. P. and Foster, D. W. (1977) A possible role for malonyl-CoA in the regulation of hepatic fatty acid oxidation and ketogenesis. J Clin Invest 60, 265-270

4 Bougneres, P. F., Saudubray, J. M., Marsac, C., Bernard, O., Odievre, M. and Girard, J. R. (1980) Decreased ketogenesis due to deficiency of hepatic carnitine acyl transferase. N Engl J Med 302, 123-124

5 Bonnefont, J. P., Djouadi, F., Prip-Buus, C., Gobin, S., Munnich, A. and Bastin, J. (2004) Carnitine palmitoyltransferases 1 and 2: biochemical, molecular and medical aspects. Mol Aspects Med 25, 495-520

6 Fromenty, B. and Pessayre, D. (1995) Inhibition of mitochondrial beta-oxidation as a mechanism of hepatotoxicity. Pharmacol Ther 67, 101-154

7 Grefhorst, A., Hoekstra, J., Derks, T. G., Ouwens, D. M., Baller, J. F., Havinga, R., Havekes, L. M., Romijn, J. A. and Kuipers, F. (2005) Acute hepatic steatosis in mice by blocking beta-oxidation does not reduce insulin sensitivity of very-low-density lipoprotein production. Am J Physiol Gastrointest Liver Physiol 289, G592-598

8 Vickers, A. E., Bentley, P. and Fisher, R. L. (2006) Consequences of mitochondrial injury induced by pharmaceutical fatty acid oxidation inhibitors is characterized in human and rat liver slices. Toxicol In Vitro 20, 1173-1182

9 Stefanovic-Racic, M., Perdomo, G., Mantell, B. S., Sipula, I. J., Brown, N. F. and O'Doherty, R. M. (2008) A moderate increase in carnitine palmitoyltransferase 1a 
602

603

604

605

activity is sufficient to substantially reduce hepatic triglyceride levels. Am J Physiol Endocrinol Metab 294, E969-977

10 Prip-Buus, C., Perdereau, D., Foufelle, F., Maury, J., Ferre, P. and Girard, J. (1995) Induction of fatty-acid-synthase gene expression by glucose in primary culture of rat hepatocytes. Dependency upon glucokinase activity. Eur J Biochem 230, 309-315

11 Ontko, J. A. and Johns, M. L. (1980) Evaluation of malonyl-CoA in the regulation of long-chain fatty acid oxidation in the liver. Evidence for an unidentified regulatory component of the system. Biochem J 192, 959-962

12 Bremer, J. (1981) The effect of fasting on the activity of liver carnitine palmitoyltransferase and its inhibition by malonyl-CoA. Biochim Biophys Acta 665, 628-631

13 Grantham, B. D. and Zammit, V. A. (1986) Restoration of the properties of carnitine palmitoyltransferase I in liver mitochondria during re-feeding of starved rats. Biochem J 239, 485-488

14 Grantham, B. D. and Zammit, V. A. (1988) Role of carnitine palmitoyltransferase I in the regulation of hepatic ketogenesis during the onset and reversal of chronic diabetes. Biochem J 249, 409-414

15 Faye, A., Borthwick, K., Esnous, C., Price, N. T., Gobin, S., Jackson, V. N., Zammit, V. A., Girard, J. and Prip-Buus, C. (2005) Demonstration of N- and C-terminal domain intramolecular interactions in rat liver carnitine palmitoyltransferase 1 that determine its degree of malonyl-CoA sensitivity. Biochem J 387, 67-76

16 Prip-Buus, C., Cohen, I., Kohl, C., Esser, V., McGarry, J. D. and Girard, J. (1998) Topological and functional analysis of the rat liver carnitine palmitoyltransferase 1 expressed in Saccharomyces cerevisiae. FEBS Lett 429, 173-178

17 Laemmli, U. K. (1970) Cleavage of structural proteins during the assembly of the head of bacteriophage T4. Nature 227, 680-685

18 Lowry, O. H., Rosebrough, N. J., Lewis Farr, A. and Randall, R. J. (1951) J. Biol. Chem. 193, 265-275

19 Prip-Buus, C., Pegorier, J. P., Duee, P. H., Kohl, C. and Girard, J. (1990) Evidence that the sensitivity of carnitine palmitoyltransferase I to inhibition by malonyl-CoA is an important site of regulation of hepatic fatty acid oxidation in the fetal and newborn rabbit. Perinatal development and effects of pancreatic hormones in cultured rabbit hepatocytes. Biochem J 269, 409-415

20 Ferre, P., Pegorier, J. P., Williamson, D. H. and Girard, J. (1979) Interactions in vivo between oxidation of non-esterified fatty acids and gluconeogenesis in the newborn rat. Biochem J 182, 593-598

21 Guillet-Deniau, I., Pichard, A. L., Kone, A., Esnous, C., Nieruchalski, M., Girard, J. and Prip-Buus, C. (2004) Glucose induces de novo lipogenesis in rat muscle satellite cells through a sterol-regulatory-element-binding-protein-1c-dependent pathway. J Cell Sci 117, 1937-1944

22 Fujioka, T., Tsujita, Y. and Shimotsu, H. (1997) Induction of fatty acid synthesis by pravastatin sodium in rat liver and primary hepatocytes. Eur J Pharmacol 328, 235-239

23 Halestrap, A. P. and Denton, R. M. (1973) Insulin and the regulation of adipose tissue acetyl-coenzyme A carboxylase. Biochem J 132, 509-517

24 Antinozzi, P. A., Segall, L., Prentki, M., McGarry, J. D. and Newgard, C. B. (1998) Molecular or pharmacologic perturbation of the link between glucose and lipid metabolism is without effect on glucose-stimulated insulin secretion. A re-evaluation of the long-chain acyl-CoA hypothesis. J Biol Chem 273, 16146-16154

25 Panek, E., Cook, G. A. and Cornell, N. W. (1977) Inhibition by 5-(tetradecyloxy)-2furoic acid of fatty acid and cholesterol synthesis in isolated rat hepatocytes. Lipids 
606

607

608

609

610

611

612

613

614

615

616

617

618

619

620

621

622

623

624

625

626

627

628

629

630

631

632

633

634

635

636

637

638

639

640

641

642

643

644

645

646

647

648

649

650

651

652

653

654

655
12, 814-818

26 Morillas, M., Gomez-Puertas, P., Bentebibel, A., Selles, E., Casals, N., Valencia, A., Hegardt, F. G., Asins, G. and Serra, D. (2003) Identification of conserved amino acid residues in rat liver carnitine palmitoyltransferase I critical for malonyl-CoA inhibition. Mutation of methionine 593 abolishes malonyl-CoA inhibition. J Biol Chem 278, 9058-9063

27 Herrero, L., Rubi, B., Sebastian, D., Serra, D., Asins, G., Maechler, P., Prentki, M. and Hegardt, F. G. (2005) Alteration of the malonyl-CoA/carnitine palmitoyltransferase I interaction in the beta-cell impairs glucose-induced insulin secretion. Diabetes 54, $462-471$

28 Sebastian, D., Herrero, L., Serra, D., Asins, G. and Hegardt, F. G. (2007) CPT I overexpression protects L6E9 muscle cells from fatty acid-induced insulin resistance. Am J Physiol Endocrinol Metab 292, E677-686

29 Perdomo, G., Commerford, S. R., Richard, A. M., Adams, S. H., Corkey, B. E., O'Doherty, R. M. and Brown, N. F. (2004) Increased beta-oxidation in muscle cells enhances insulin-stimulated glucose metabolism and protects against fatty acidinduced insulin resistance despite intramyocellular lipid accumulation. J Biol Chem 279, 27177-27186

30 Linden, D., William-Olsson, L., Ahnmark, A., Ekroos, K., Hallberg, C., Sjogren, H. P., Becker, B., Svensson, L., Clapham, J. C., Oscarsson, J. and Schreyer, S. (2006) Liver-directed overexpression of mitochondrial glycerol-3-phosphate acyltransferase results in hepatic steatosis, increased triacylglycerol secretion and reduced fatty acid oxidation. Faseb J 20, 434-443

31 Linden, D., William-Olsson, L., Rhedin, M., Asztely, A. K., Clapham, J. C. and Schreyer, S. (2004) Overexpression of mitochondrial GPAT in rat hepatocytes leads to decreased fatty acid oxidation and increased glycerolipid biosynthesis. J Lipid Res 45, 1279-1288

32 Prip-Buus, C., Bouthillier-Voisin, A. C., Kohl, C., Demaugre, F., Girard, J. and Pegorier, J. P. (1992) Evidence for an impaired long-chain fatty acid oxidation and ketogenesis in Fao hepatoma cells. Eur J Biochem 209, 291-298

33 Monetti, M., Levin, M. C., Watt, M. J., Sajan, M. P., Marmor, S., Hubbard, B. K., Stevens, R. D., Bain, J. R., Newgard, C. B., Farese, R. V., Sr., Hevener, A. L. and Farese, R. V., Jr. (2007) Dissociation of hepatic steatosis and insulin resistance in mice overexpressing DGAT in the liver. Cell Metab 6, 69-78

34 Randle, P. J. (1998) Regulatory interactions between lipids and carbohydrates: the glucose fatty acid cycle after 35 years. Diabetes Metab Rev 14, 263-283

35 Fromenty, B., Robin, M. A., Igoudjil, A., Mansouri, A. and Pessayre, D. (2004) The ins and outs of mitochondrial dysfunction in NASH. Diabetes Metab 30, 121-138

36 Savage, D. B., Choi, C. S., Samuel, V. T., Liu, Z. X., Zhang, D., Wang, A., Zhang, X. M., Cline, G. W., Yu, X. X., Geisler, J. G., Bhanot, S., Monia, B. P. and Shulman, G. I. (2006) Reversal of diet-induced hepatic steatosis and hepatic insulin resistance by antisense oligonucleotide inhibitors of acetyl-CoA carboxylases 1 and 2. J Clin Invest 116, 817-824

37 Dentin, R., Benhamed, F., Hainault, I., Fauveau, V., Foufelle, F., Dyck, J. R., Girard, J. and Postic, C. (2006) Liver-specific inhibition of ChREBP improves hepatic steatosis and insulin resistance in ob/ob mice. Diabetes 55, 2159-2170

38 An, J., Muoio, D. M., Shiota, M., Fujimoto, Y., Cline, G. W., Shulman, G. I., Koves, T. R., Stevens, R., Millington, D. and Newgard, C. B. (2004) Hepatic expression of malonyl-CoA decarboxylase reverses muscle, liver and whole-animal insulin resistance. Nat Med 10, 268-274 


\section{FOOTNOTES}

659 Abbreviations used: CPT1, carnitine palmitoyltransferase 1; CPT1A, liver isoform of CPT1; 660 LCFA, long-chain fatty acid; LC-CoA, long-chain acyl-CoA; ACC, acetyl-CoA carboxylase; 661 FAS, fatty acid synthase; TG, triglyceride; VLDL, very-low-density lipoprotein; TOFA, 5662 (Tetradecyloxy)-2-furoic acid; Ad, adenovirus; $\beta$ gal, $\beta$-galactosidase; CPT1wt, wild-type rat 663 CPT1A; CPT1mt, mutant CPT1AM593S; ip, infectious particules; CPT2, carnitine 664 palmitoyltransferase 2; ASP, acid-soluble product; PL, phospholipids; MCD, malonyl-CoA 665 decarboxylase.

666

667

668

669

\section{LEGENDS TO FIGURES}

Figure 1. Mitochondrial localization of the overexpressed CPT1wt and CPT1mt proteins in cultured rat hepatocytes

672 Primary rat hepatocytes were infected with either $0,2.5$ or $5 \mathrm{ip} /$ cell of the indicated

673

674

675

676

677

678

679

680

681

682

683

684

685

686

687

688

689

690

691

692

693

694

695

696

697

698

699

700

701

702

703

704 adenovirus and cultured for $40 \mathrm{~h}$ in $5 \mathrm{mM}$ glucose. (a) Immunoblot analysis of cellular extracts $(30 \mu \mathrm{g}$ of protein) using specific antibodies for $\beta$-galactosidase ( $\beta$ gal $)$, CPT1A and CPT2. Western blots are representative of four independent experiments performed in duplicates. (b) Immunofluorescence analysis detection of the overexpressed CPT1 proteins. Hepatocytes were double-immunostained with antibodies raised against cytochrome c (Cyt c) and CPT1A. The right panels (merge) represent the overlay of the two left panels. Colocalization of cytochrome $\mathrm{c}$ and CPT1A appears as yellow spots reflecting the merge of red (Cyt c) and green (CPT1A) fluorescence. Hoechst 33342 was used to stain the hepatocyte nuclei. The bar represents $50 \mu \mathrm{m}$.

Figure 2. Effects of CPT1wt and CPT1mt overexpression on CPT1A protein level and activity (a) and malonyl-CoA sensitivity (b) in mitochondria isolated from infected hepatocytes

Mitochondria were isolated $40 \mathrm{~h}$ after infection of hepatocytes with $5 \mathrm{ip} /$ cell of the indicated adenovirus. (a) Immunoblot analysis of mitochondrial protein using specific antibodies for CPT1A and CPT2. Western blots are representative of three independent experiments with mitochondria isolated from separate hepatocytes cultures. CPT1A and CPT2 activities were measured in either intact (CPT1A) or solubilized (CPT2) mitochondria. Results are mean \pm S.E.M. of three independent experiments with separate isolated mitochondria. (b) MalonylCoA sensitivity of CPT1A was measured in intact isolated mitochondria. Results are mean \pm S.E.M. of two independent experiments with separate isolated mitochondria. ${ }^{*} P<0.01$ vs. Ad- $\beta$ gal; $\# P<0.01$.

Figure 3. CPT1wt and CPT1mt overexpression counteracts the glucose/insulin effects on $\left[1-{ }^{14} \mathrm{C}\right]$ oleate metabolism

Sixteen hours after infection of hepatocytes with $5 \mathrm{ip} /$ cell of the indicated adenovirus, cells were cultured for $24 \mathrm{~h}$ in the presence of either $5 \mathrm{mM}$ glucose (G5) or $20 \mathrm{mM}$ glucose plus 10 $\mathrm{nM}$ insulin $(\mathrm{G} 20+\mathrm{ins})$. During the last $2 \mathrm{~h}$ of culture, $0.3 \mathrm{mM}\left[1-{ }^{14} \mathrm{C}\right]$ oleate bound to $1 \%$ defatted BSA was added. Oleate oxidation into $\mathrm{CO}_{2}$ (a) and acid-soluble products (ASP) (b), and oleate esterification into cellular triglycerides (TG) (c) and phospholipids (PL) (d) were measured at the end of the $2 \mathrm{~h}$-incubation period. (e) Metabolic orientation of oleate as a percentage of the total nmol of metabolized $\left[1-{ }^{14} \mathrm{C}\right]$ oleate. (f) $\left[1-{ }^{14} \mathrm{C}\right]$ Oleate incorporation into 
705

706

707

708

709

710

711

712

713

714

715

716

717

718

719

720

721

722

723

724

725

726

727

728

729

730

731

732

733

734

735

736

737

738

739

740

741

742

743

744

745

746

747

748

very-low-density lipoprotein (VLDL) secreted in the culture medium during the $2 \mathrm{~h}$ incubation period. Results are mean \pm S.E.M. of duplicate flasks from four independent experiments. $* P<0.05$ and $* * P<0.01$ vs. Ad- $\beta$ gal; $\# P<0.05$.

Figure 4. CPT1mt overexpression decreases the glucose/insulin-induced TG accumulation in cultured hepatocytes

Sixteen hours after infection with $5 \mathrm{ip} /$ cell of the indicated adenovirus, hepatocytes were cultured for $24 \mathrm{~h}$ in the presence of either G5 or G20 + ins. (a) Oil red O staining of hepatocytes to detect neutral lipid droplets. Oleate $(0.3 \mathrm{mM})$ was added during the last $2 \mathrm{~h}$ of culture. Original magnification, X 200. (b) Measurement of cellular TG content. When indicated, $0.3 \mathrm{mM}$ oleate was added during the last $2 \mathrm{~h}$ of culture. Results are mean \pm S.E.M. of duplicate flasks from three independent experiments. ${ }^{*} P<0.05$ and $* * P<0.01$ vs. Ad$\beta$ gal in $\mathrm{G} 20+$ ins; $\# P<0.05$.

Figure 5. CPT1wt and CPT1mt overexpression does not alter the glucose/insulin effects on $\left[\mathrm{U}-{ }^{14} \mathrm{C}\right]$ glucose metabolism

Sixteen hours after infection with $5 \mathrm{ip} /$ cell of the indicated adenovirus, hepatocytes were cultured for $24 \mathrm{~h}$ in the presence of either G5 or G20 + ins. During the last $2 \mathrm{~h}$, a tracer amount of [ $\left.\mathrm{U}_{-}{ }^{14} \mathrm{C}\right]$ glucose was added in the absence or in the presence of $0.3 \mathrm{mM}$ oleate. The medium was then collected to measure $\left[\mathrm{U}_{-}{ }^{14} \mathrm{C}\right]$ glucose oxidation into $\mathrm{CO}_{2}$ (a). Cells were washed and scrapped to measure incorporation of $\left[\mathrm{U}_{-}^{14} \mathrm{C}\right]$ glucose into glycogen (b). Results are mean \pm S.E.M. of duplicate flasks from five independent experiments. $\S P<0.01 \mathrm{vs}$. G5.

Figure 6. CPT1wt and CPT1mt overexpression allows oxidation of de novo synthetised LCFA

Sixteen hours after infection with $5 \mathrm{ip} / \mathrm{cell}$ of the indicated adenovirus, hepatocytes were cultured for $24 \mathrm{~h}$ in the presence of either G5 or G20+ ins, in the absence or presence of 200 $\mu \mathrm{M}$ 5-(Tetradecyloxy)-2-furoic acid (TOFA). When indicated, $0.3 \mathrm{mM}$ oleate was added during the last $2 \mathrm{~h}$ of culture. (a) The medium was collected to measure $\beta$-hydroxybutyrate production. (b) The lipogenesis rate from $5 \mathrm{mM}\left[1-{ }^{14} \mathrm{C}\right]$ acetate was measured, in the absence of exogenous oleate, in uninfected cells during the last $2 \mathrm{~h}$ of culture. (c) Measurement of cellular TG content. Results are mean \pm S.E.M. of duplicate flasks from three to seven independent experiments. $† P<0.01$ vs. without oleate; $* P<0.05$ and $* * P<0.01$ vs. Ad$\beta$ gal in $\mathrm{G} 20+$ ins; $\# P<0.01$

Figure 7. Importance of the malonyl-CoA/CPT1A partnership in the liver

In physiological situations, inhibition of CPT1A by malonyl-CoA preserves liver cells from wasting energy by preventing ß-oxidation of long-chain fatty acids (LCFA) de novo synthetised from glucose. Indeed, retrieving CPT1A from malonyl-CoA inhibition (expression of a malonyl-CoA-insensitive CPT1A i.e. CPT1mt) induces a metabolic switch toward B-oxidation of LCFA independently of their origin (exogenous or de novo synthetised), and prevents triglycerides (TG) accumulation in liver cells. Therefore, malonyl$\mathrm{CoA} / \mathrm{CPT} 1 \mathrm{~A}$ partnership greatly contributes to the physiological function of the liver to convert excess dietary carbohydrates into TG. 


\section{Figure 1}

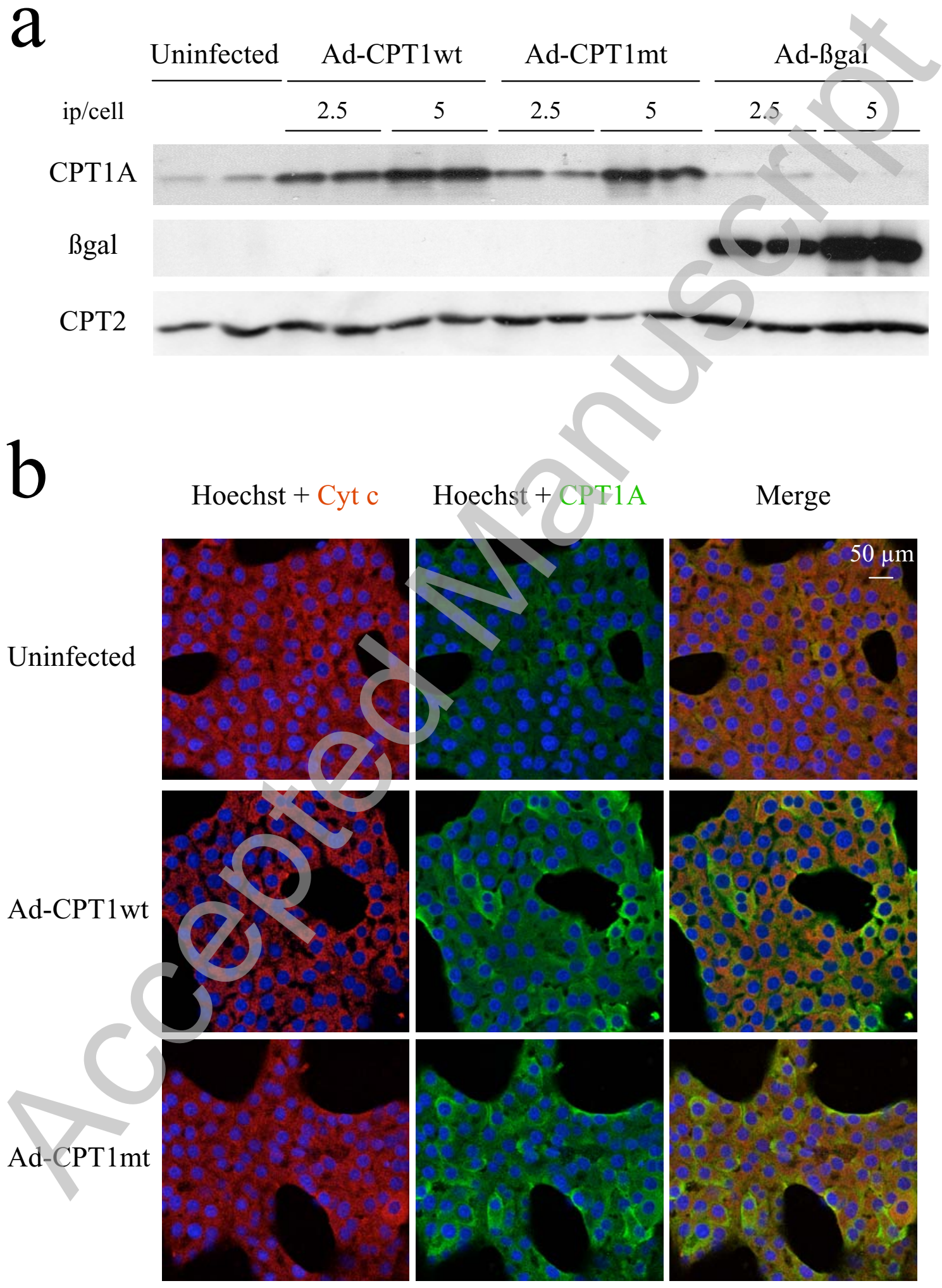


Figure 2

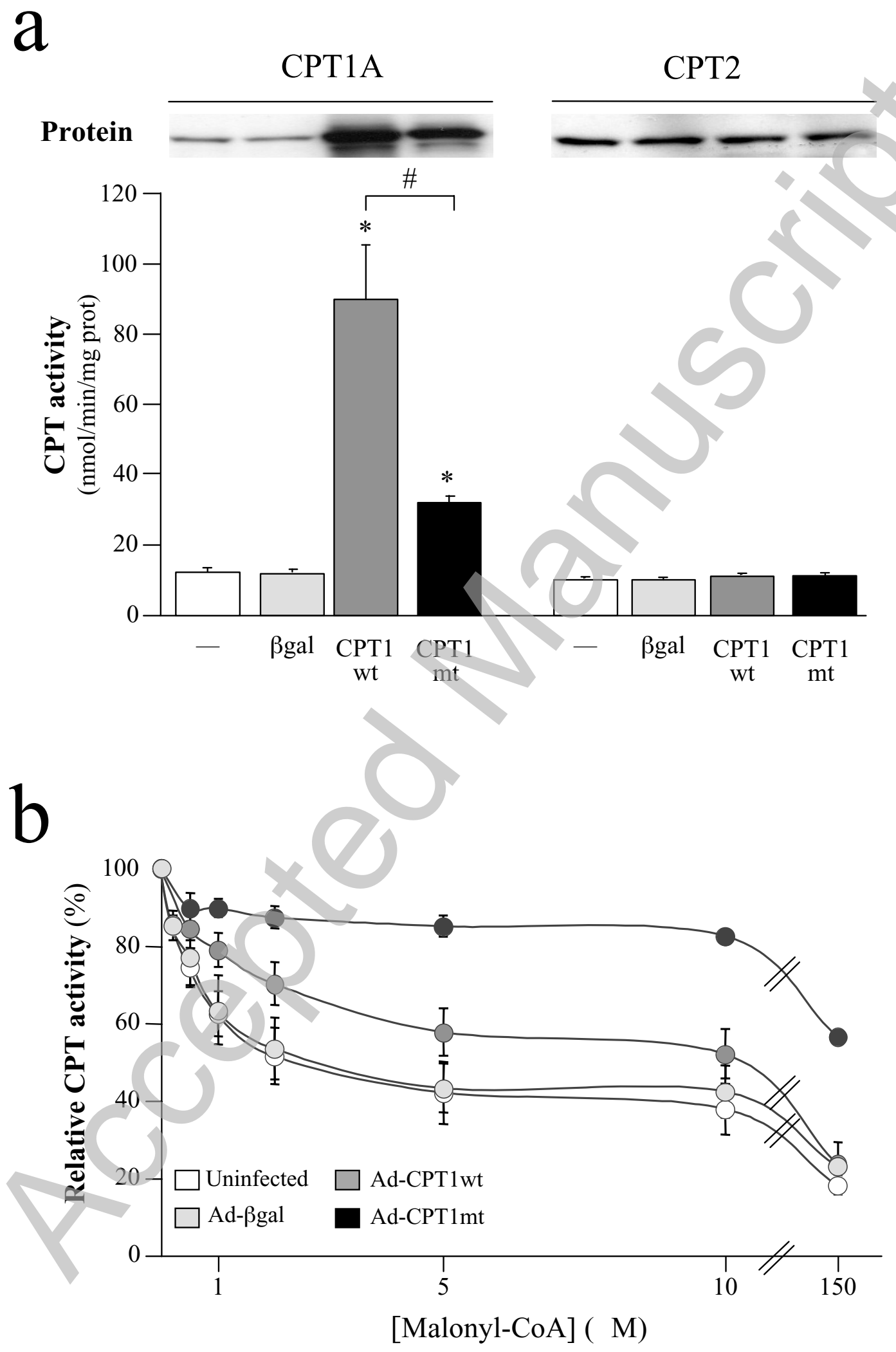


Figure 3

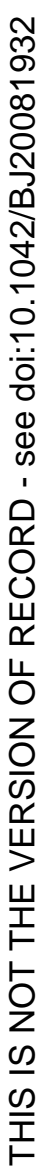

a

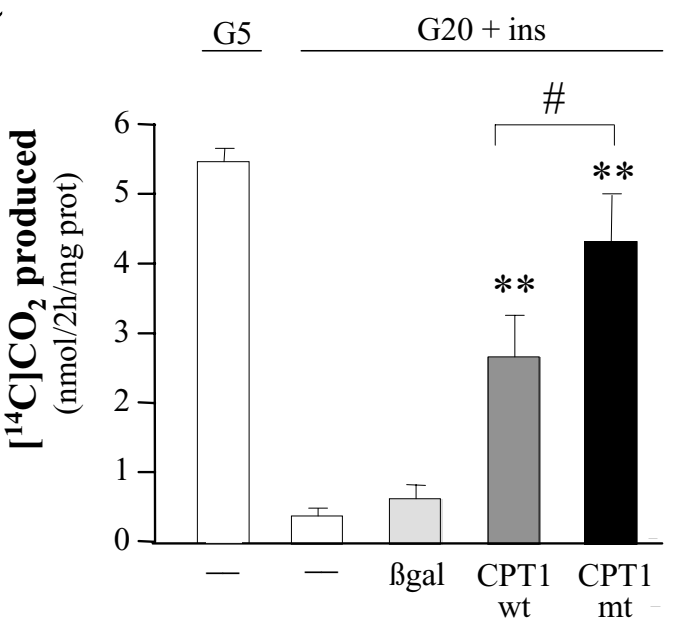

C

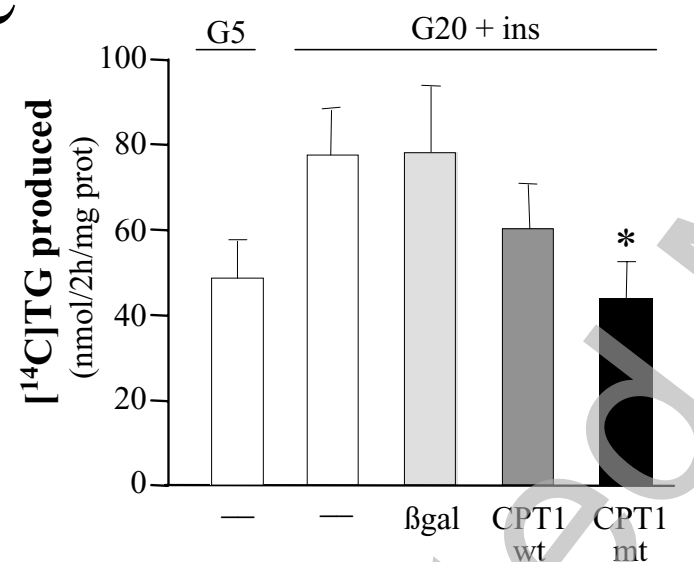

e

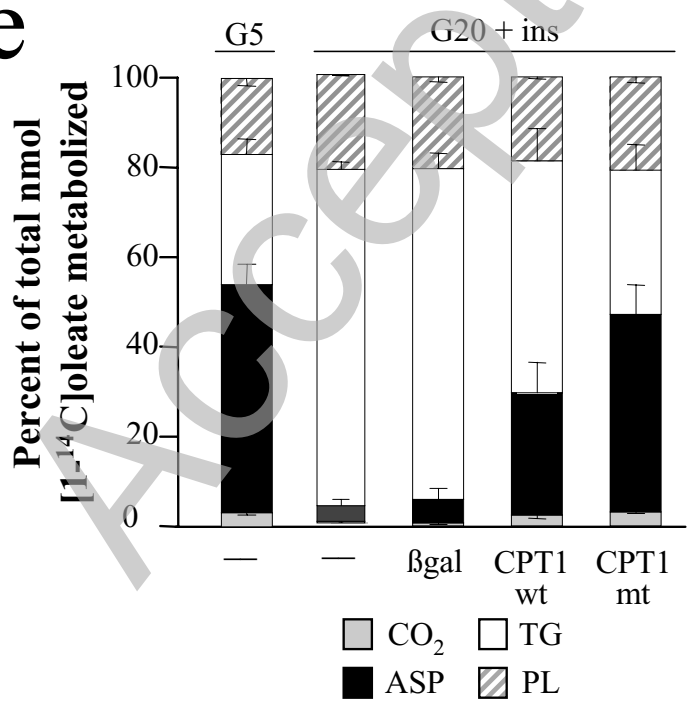

b

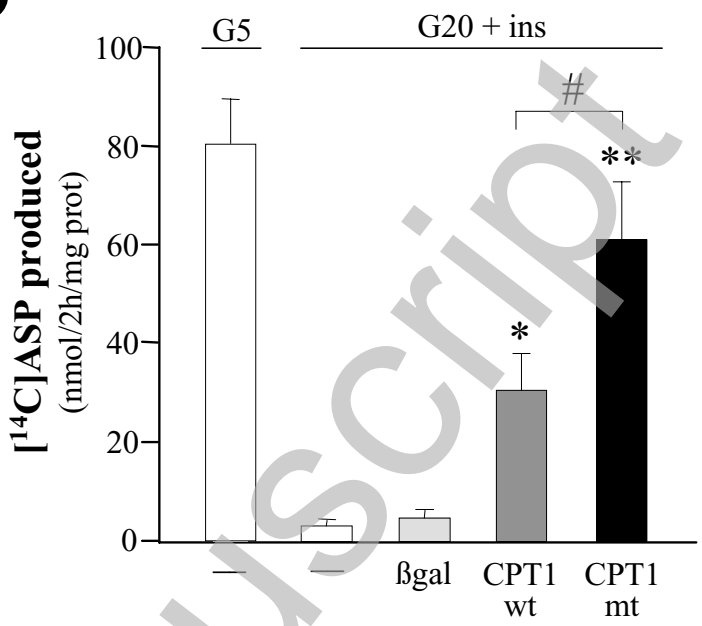

d

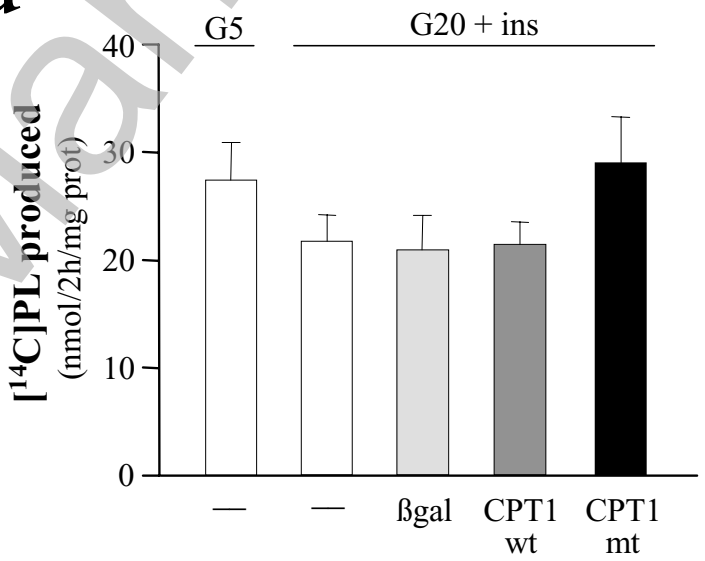

$\mathrm{f}$

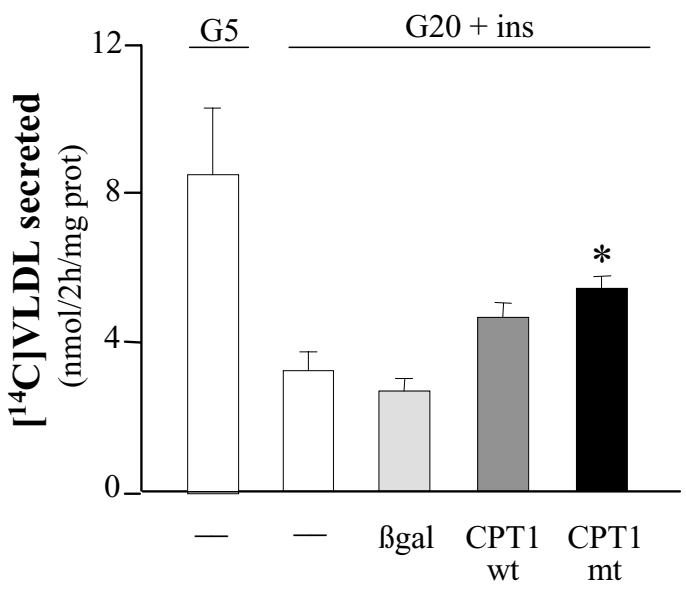


Figure 4

a
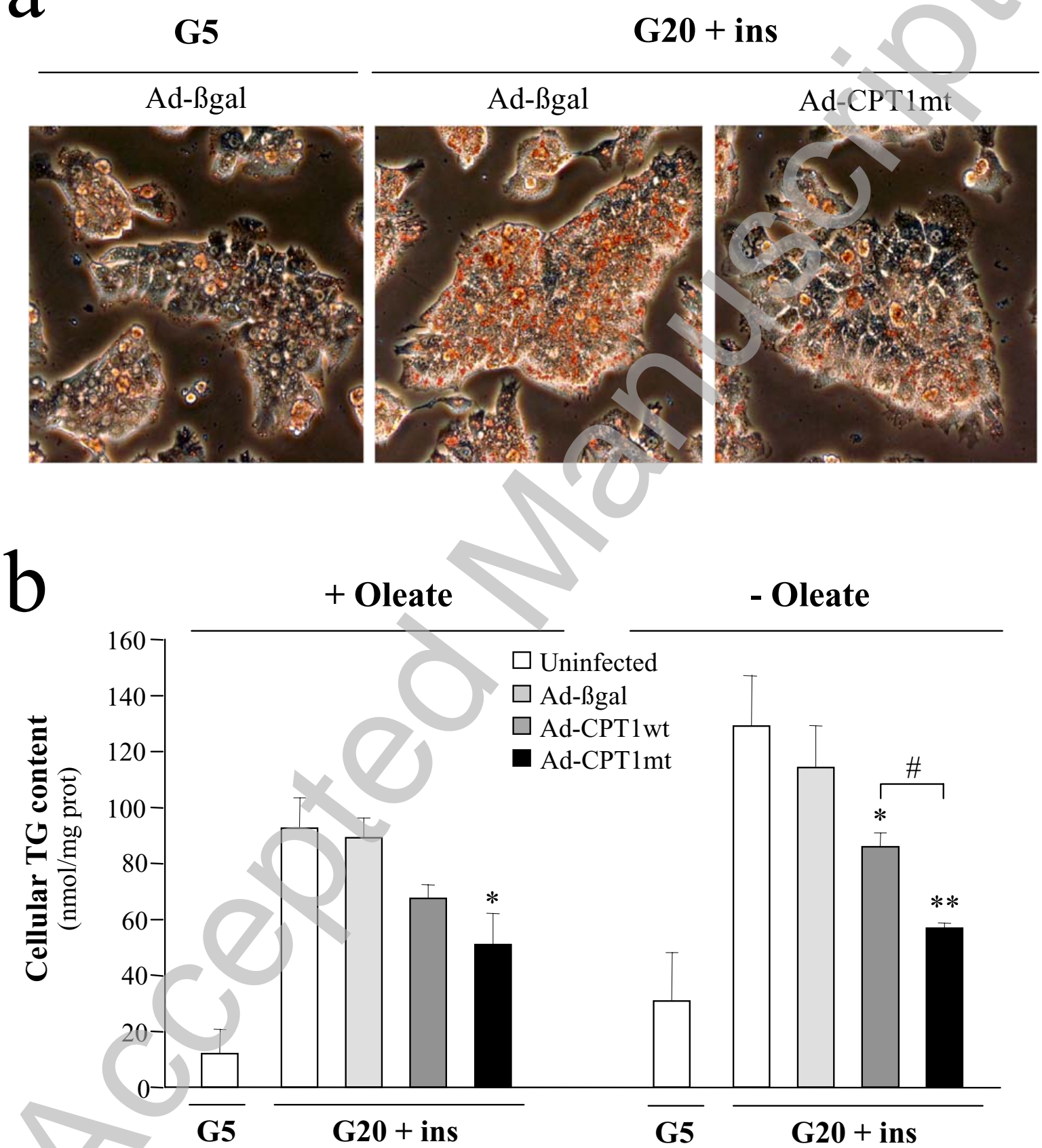
Figure 5

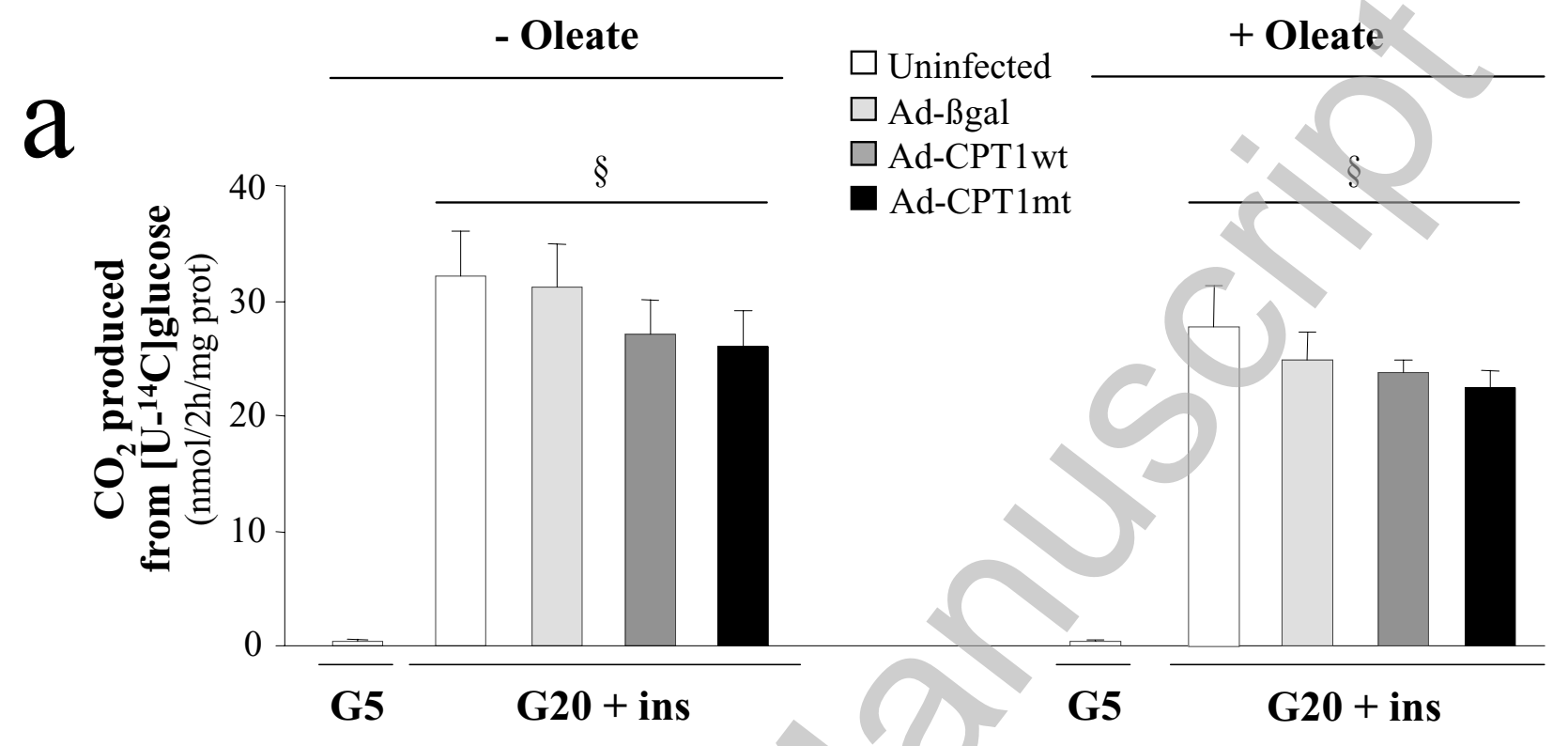

b

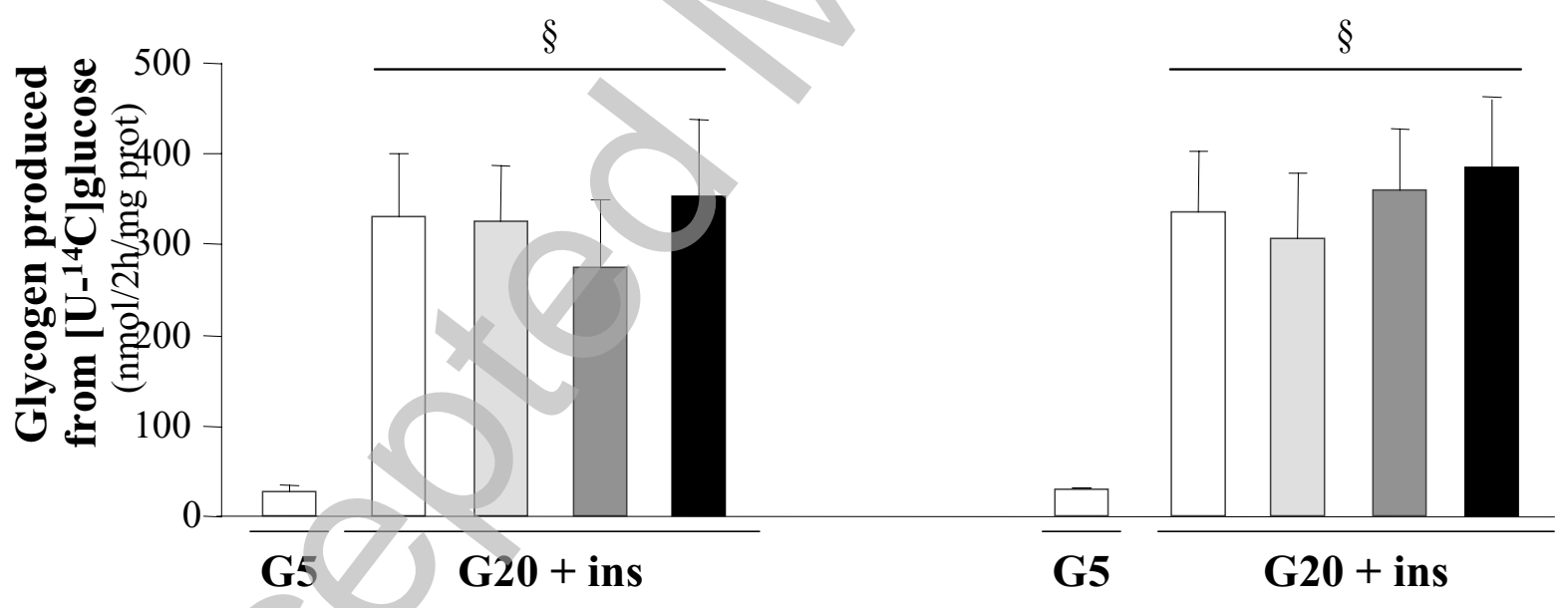


Figure 6

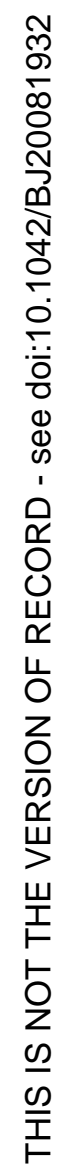

a

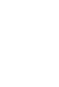

- Oleate

$\overline{\mathrm{G} 5} \mathrm{G} 20+\mathrm{ins} \quad \mathrm{G} 20+\mathrm{ins}+\mathrm{TOFA}$

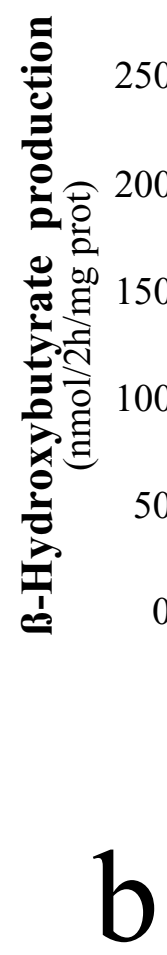

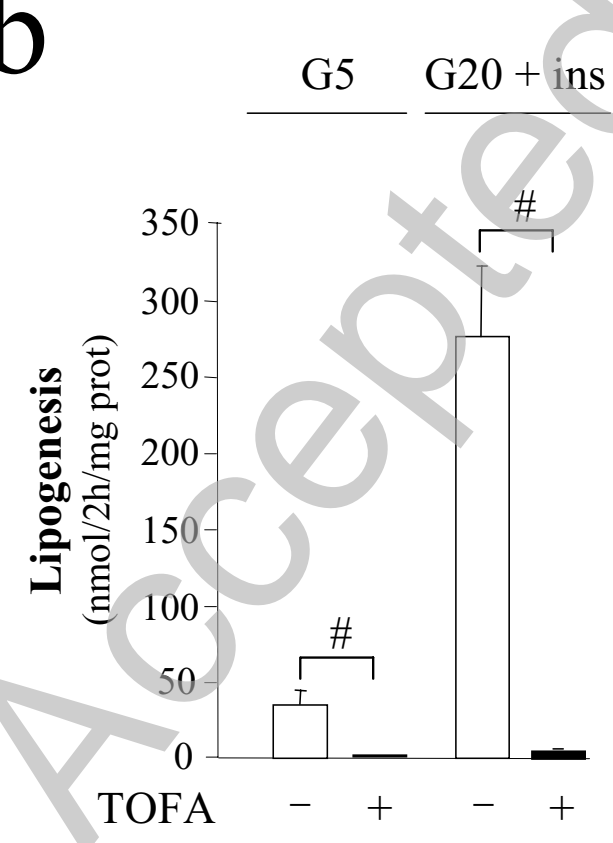

C

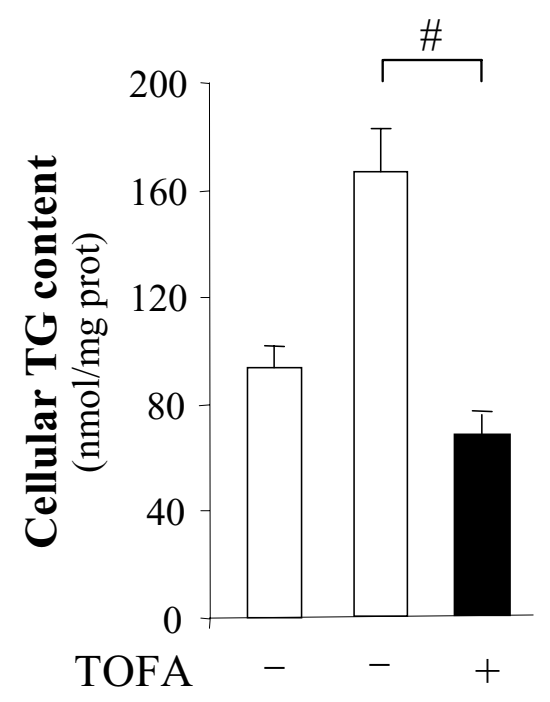



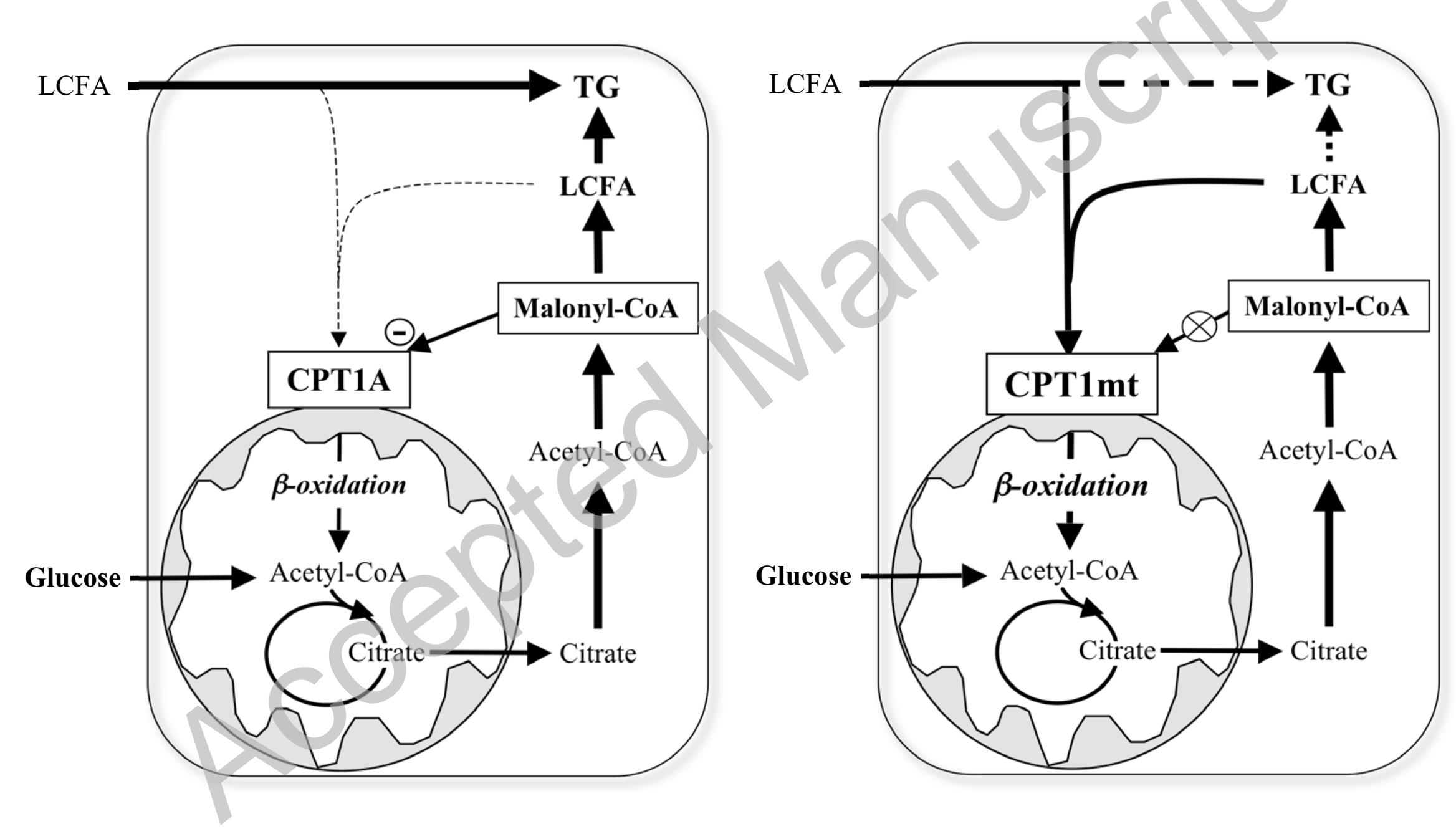